\title{
Prognostic value of bcl-2 expression in patients with operable carcinoma of the uterine cervix
}

\author{
W Tjalma, J Weyler, G Goovaerts, C De Pooter, E Van Marck, P van Dam
}

Laboratory of Cancer Research and Clinical Oncology, University of Antwerp, Antwerp, Belgium W Tjalma C De Pooter

$P$ van Dam

\section{Department of} Epidemiology and Community Medicine, University of Antwerp J Weyler

\section{Department of} Pathology, General Hospital Saint Camillus/Saint Augustinus Hospital, Antwerp, Belgium G Goovaerts

\section{Department of} Obstetrics and Gynaecology, General Hospital Saint Camillus/Saint Augustinus Hospital W Tjalma

Department of Pathology, Antwerp University Hospital, Antwerp, Belgium

E Van Marck

\section{Department of} Obstetrics and Gynaecology, Antwerp University Hospital $P$ van Dam

Correspondence to: Professor P van Dam, Department of Obstetrics and Gynaecology, Division of Gynaecological Oncology, Antwerp University Hospital Wilrijkstraat 10, B 2650

Edegem, Belgium.

\begin{abstract}
Aim-To evaluate the patterns of bcl-2 expression in early stage cervical carcinoma; to compare bcl-2 expression with clinicopathological findings; and to assess its prognostic value.

Methods-Wertheim radical hysterectomy specimens from 76 patients (FIGO stages Ia-IIb) with untreated nonmetastatic invasive cervical carcinoma were studied. Expression of bcl-2 was detected immunohistochemically using a monoclonal antibody. A tumour was regarded as positive when more than $5 \%$ of the neoplastic cells exhibited bcl-2 immunoreactivity.

Results-Forty eight (63\%) cervical carcinomas were scored as bcl-2 positive and 28 (37\%) as bcl-2 negative. Most tumours showed heterogeneous cytoplasmic staining. Bcl-2 immunoreactivity did not correlate with tumour histology, tumour stage, presence of lymph node metastases, or involvement of the lymphovascular space. The five year survival rate for patients with bcl-2 negative tumours was $34 \%$ and was $71 \%$ for patients with bcl-2 positive tumours. On multiple regression analysis (Cox proportional hazards model), bcl-2 expression and vascular permeation were independent predictors of overall survival.

Conclusions-Bcl-2 expression seems to be associated with less aggressive behaviour in early stage cervical carcinoma. The transition to bcl-2 independence may play an important role in tumour progression.

(F Clin Pathol 1997;50:33-36)
\end{abstract}

Keywords: bcl-2; cervical cancer; immunohistochemistry; prognosis.

In recent years it has become clear that carcinogenesis cannot only be explained by the increased stimulation of cell growth, but can also be caused by loss of growth suppression, changes in programmed cell death (apoptosis) and alterations in immune surveillance. Apoptosis and mitosis are closely inter-related, and there is some evidence that both in normal and neoplastic tissues very complex multifactorial control mechanisms (such as p53, c-myc, c-fos, bax, bcl-2 expression) determine whether cells will progress to mitosis or apoptosis. ${ }^{1}$ The regulation of apoptosis is central to morphogenesis during fetal development and to the maintenance of tissue homeostasis during adulthood and seems to be of importance for neoplastic transformation in some organs. One of the most important genes in this process is bcl-2. This gene is unique among proto-oncogenes because it has the ability to block programmed cell death without promoting cell proliferation. ${ }^{2}$ The 25 kilodalton bcl- 2 protein is located at the mitochondrial outer membrane, in the nuclear envelope, plasma membrane, endoplasmic reticulum, and in chromosomes. ${ }^{3-7} \mathrm{Bcl}-2$ expression was first detected in folliculair lymphomas with a balanced chromosomal translocation $t(14 ; 18)(\mathrm{q} 32 ; 21)$, but later bcl-2 overexpression was also found in lymphomas and epithelial malignancies lacking this translocation.

When overexpressed, bcl-2 produces dramatic extension of cell survival. Overexpression of bcl-2 is associated in a number of tumours with less aggresive malignant behaviour. The aim of the present retrospective study was to elucidate the functional role of bcl-2 in carcinoma of the uterine cervix using archival paraffin wax embedded tissue specimens and to compare bcl-2 expression with clinicopathological findings.

\section{Methods}

PATIENTS AND SPECIMENS

Wertheim radical hysterectomy specimens from 76 patients with untreated invasive carcinoma of the uterine cervix were studied. All operations were performed between 1979 and 1995 in the Departments of Obstetrics and Gynaecology of the Antwerp University and of the General Hospital Saint Camillus/Saint Augustinus. Patients underwent a preoperative blood test (full blood count, clotting parameters, electrolytes, liver function tests, urea creatinine, carcinoembryonic antigen), chest $x$ ray, computed tomography scan of the abdomen and pelvis, and an examination under anaesthesia (including cystoscopy and sigmoidoscopy). The median age of the patients at the time of diagnosis was 53 years (range 24-82). Tumours were staged according to FIGO criteria. ${ }^{8}$ Six patients had FIGO stage Ia tumours, 37 stage Ib, 29 stage IIa, and four stage IIb. Histological subtyping of tumours followed WHO guidelines (1981).

Postoperatively, all 25 patients with lymph node metastases and the six patients with negative lymph nodes but evidence of lymphovascular space involvement received adjuvant external field pelvic radiotherapy. The doses varied from 1.5 to 2 Gray daily, given in a four field technique, with a maximum of 50 Gray in total. Follow up examinations, consist- 
Table 1 Relation between classic prognostic factors and bcl-2 cytoplasmic immunoreactivity

\begin{tabular}{|c|c|c|c|}
\hline Classic prognostic factors & $n$ & bcl-2 positivity (n (\%)) & $p$ value ( $\chi^{2}$ test) \\
\hline \multicolumn{4}{|l|}{ Histology } \\
\hline Squamous carcinoma & 59 & $38(64)$ & NS \\
\hline Adenosquamous carcinoma & 6 & $2(33)$ & \\
\hline Adenocarcinoma & 7 & $5(71)$ & \\
\hline Other & 4 & $3(75)$ & \\
\hline \multicolumn{4}{|l|}{ Tumour grade } \\
\hline I & 15 & $11(73)$ & NS \\
\hline II & 35 & $21(60)$ & \\
\hline III & 26 & $16(62)$ & \\
\hline \multicolumn{4}{|l|}{ Lymphatic permeation } \\
\hline Absent & 40 & $28(70)$ & NS \\
\hline Present & 36 & $20(56)$ & \\
\hline \multicolumn{4}{|l|}{ Vascular permeation } \\
\hline Absent & 51 & $34(67)$ & NS \\
\hline Present & 25 & $14(56)$ & \\
\hline Lymph node status & & & NS \\
\hline Negative & 51 & $34(67)$ & \\
\hline Positive & 25 & $14(56)$ & \\
\hline
\end{tabular}

ing of physical examination and vaginal vault smears, were done at regular intervals. Patients developing symptoms suggestive of metastatic disease were investigated further. Follow up data were available in all 76 patients. Median observation time was 39 months (range four to 203).

\section{bcl-2 IMMUNOSTAINING}

Sections, $5 \mu \mathrm{m}$ thick, were cut from the formalin fixed, paraffin wax embedded tumour specimens and deparaffinised routinely. They were then treated with $0.3 \%$ hydrogen peroxide to block endogenous peroxidase activity. The slides were placed in a 0.01 citrate buffer and heated in the microwave oven at $700 \mathrm{~W}$ for $2 \times 5$ minutes. $\mathrm{Bcl}-2$ expression was detected by using a standard immunohistochemical procedure, incubating the slides for 30 minutes with monoclonal mouse anti-human bcl-2 (clone 124, isotype IgG1; Dako, Glostrup, Denmark) diluted 1 in 40 in phosphate buffered saline (PBS) containing 1\% bovine serum albumin (BSA). After three washes with PBS, the slides were incubated with secondary biotinylated rabbit anti-mouse antibodies (Dako) (diluted 1 in 350) for 30 minutes. Then they were exposed to avidin biotin peroxidase complex (Dako). Diaminobezidine tetrachlo-

Figure 1 Strong bcl-2 immunoreactivity in moderately differentiated squamous carcinoma of the cervix. ride (DAB) (Sigma, Poole, Dorset, UK) was used as the chromogen. Slides were counterstained with Mayer's haematoxylin. For negative controls, the primary antibody was omitted and the anti-bcl-2 antibody was substituted with an unrelated monoclonal antibody of the same concentration but directed against an unrelated antigen (monoclonal mouse antihuman CD68 antibody, isotype IgG1; Dako). Normal lymphocytes served as an internal positive control.

\section{EVALUATION OF IMMUNOSTAINING}

Bcl-2 immunostaining was scored as the percentage of cells exhibiting definite immunoreactivity in at least 500 neoplastic cells encountered in 10 randomly selected high power fields $(\times 400)$. A specimen was regarded as positive when more than $5 \%$ of the neoplastic cells showed bcl-2 immunoreactivity.

\section{STATISTICAL ANALYSIS}

All numerical data are expressed as median (range). The relation between bcl-2 immunoreactivity and the clinicopathological findings was evaluated using the $\chi^{2}$ test. To evaluate prognostic relevance, survival was computed from the time of surgery until death or date of the last follow up visit. The Kaplan-Meier method was used for univariate analysis and the log rank test was used to assess differences between groups. ${ }^{9}$ The adjusted hazard ratios and their $95 \%$ confidence intervals (CI) were calculated by means of a multiple regression analysis based on the Cox proportional hazards model. ${ }^{10}$ For all statistical analysis a $\mathrm{p}$ value $<0.05$ was regarded as statistically significant.

\section{Results}

\section{bcl-2 IMMUNOSTAINING}

Bcl-2 immunostaining was typically cytoplasmic. In normal squamous and columnar epithelium, bcl-2 immuoreactivity was observed at the basal layer and sometimes in the stroma. A heterogeneous distribution of bcl-2 expression was observed in the neoplastic cells of the tumours. There was striking intra- and intertumour variability: in some tumours only very scanty bcl-2 immunostaining was observed whereas in others large areas of bcl-2 positivity were found (fig 1). Bcl-2 immunoreactivity was rarely found at the leading edge of the tumour.

bCl-2 EXPRESSION IN RELATION TO CLINICOPATHOLOGICAL FINDINGS

Forty eight $(63 \%)$ cervical carcinomas were bcl-2 positive and 28 (37\%) were bcl-2 negative (table 1). The mean age of patients in the bcl-2 negative group was 55 years and in the bcl-2 positive group was 51 years (NS). A reflection of the quality of surgery is given by mean number of lymph nodes removed: 24 in the bcl-2 negative group and 27 in the bcl-2 positive group (NS). The relation between clinicopathological factors and bcl-2 immunoreactivity is summarised in table 1 . There was no significant relation between bcl-2 expression and tumour histology, tumour differentia- 


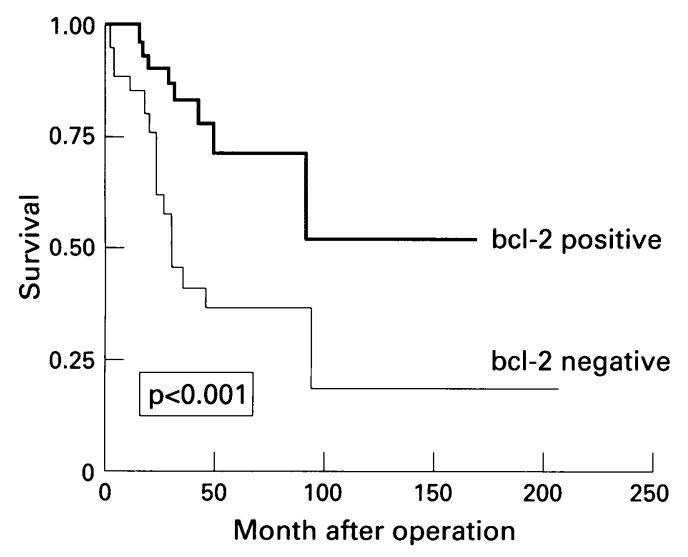

Figure 2 Kaplan-Meier curves for overall survival according to bcl-2 expression (logrank test, $p<0.001$ ) in all patients.

tion, FIGO stage, presence of lymph node metastases, or lymphovascular space involvement.

bCl-2 EXPRESSION IN RELATION TO SURVIVAL

Two women died from causes other than cervical cancer, with no evidence of disease; 42 women $(55 \%)$ were in complete remission; 34 women $(45 \%)$ had a recurrence, and 22 of the latter group ( $29 \%$ of the total study group) died of their tumours. Using univariate analysis, bcl-2 negativity ( $p<0.001)$, presence of vascular permeation $(\mathrm{p}=0.002)$ or lymphatic permeation $(p=0.014)$, and lymph node metastasis $(p=0.051)$ were significant markers of poor overall survival. The five year survival rate for the entire study population was $57 \%$ (95\% CI $40-71 \%)$. The five year survival rate for patients stratified according to FIGO stage was: $75 \%$ (95\% CI $13-96 \%)$ for stage Ia; $64 \%$ (41-80\%) for Ib; $28 \%(2-67 \%)$ for IIa; and $38 \%(1-81 \%)$ for IIb. The five year survival rate was $73 \%(46-88 \%)$ for patients without and $42 \%(22-61 \%)$ for patients with lymphatic permeation, was $69 \%(47-83 \%)$ for patients without and $33 \%(11-57 \%)$ for patients with vascular permeation, and was $66 \%(43-81 \%)$ for patients with negative and $41 \%(18-62)$ for patients with positive lymph nodes. The five year survival rate was $90 \%(47-99 \%)$ for patients with grade I tumours, $60 \%$ (35-78) for those with grade II tumours, and $39 \%$ (16-62\%) for those with grade III tumours. The five year survival rate for patients with bcl-2 negative tumours was $34 \%$ and for patients with bcl-2 positive ones was $71 \%$ (logrank test, $p<0.001$ ) (fig 2). Subgroup analysis showed that both for the lymph node negative (logrank test, $\mathrm{p}<0.001$ ) and lymph node positive groups (logrank test, $\mathrm{p}<0.001$ ), patients with bcl-2 positive tumours had a better prognosis.

The best fitting model for overall survival, according to Cox proportional hazards regression analysis (deviance of 156 , likelihood ratio on $3 \mathrm{DF}=17.751, \mathrm{p}<0.001)$, included bcl-2 expression, FIGO tumour stage and vascular permeation (table 2). Multiple regression analysis adjusted for stage and vascular permeation demonstrated a significantly shorter overall survival (adjusted hazard ratio $=0.293$
Table 2 Multivariate analysis for overall survival

\begin{tabular}{lll}
\hline & Coefficient & Standard error \\
\hline bcl-2 expression & -1.229 & 0.427 \\
FIGO stage & 0.169 & 0.335 \\
Vascular permeation & 1.155 & 0.452 \\
\hline Deviance = 156 and likelihood ratio on $3 \mathrm{DF}=17.751 ; \mathrm{p}<$ \\
0.001.
\end{tabular}

(95\% CI 0.127-0.676)) for patients with bcl-2 negative tumours compared with those with bcl-2 positive ones.

\section{Discussion}

The role of apoptosis in tumour development is currently one of the hot topics in oncology. Apoptosis is a distinct mode of programmed cell death that is responsible for the deletion of cells in normal and pathological tissues. ${ }^{2}$ Failure of tumour cells to undergo programmed cell death can result in uncontrolled accumulation of cells. In this retrospective study we found a strong relation between apoptosis, as evaluated by bcl-2 expression, and prognosis in patients with cervical carcinoma. No relation was found between bcl-2 overexpression and clinicopathological factors. However, this may be because of the limited number of patients studied, as there was a trend towards a positive correlation between bcl-2 expression and lymph node status or lymphatic tumour permeation.

$\mathrm{Bcl}-2$ overexpression has been reported to be a marker of good prognosis in patients with $B$ cell lymphomas, ${ }^{11}$ non-small cell lung cancer, ${ }^{12}$ colorectal carcinomas, ${ }^{13}$ breast cancer, ${ }^{14}$ and ovarian carcinoma. ${ }^{15}$ To the best of our knowledge this is the first study demonstrating that bcl-2 is an independent prognostic factor in patients with early carcinoma of the uterine cervix. Levine et $a l^{16}$ stated that spontaneous apoptosis in cervical tumours may be a predictor of response to radiotherapy. In the present study bcl-2 expression was an important independent prognostic factor in patients who were treated primarily by radical surgery. Subgroup analysis revealed that both in patients who received adjuvant radiotherapy (lymph node positive) or in patients who were only treated surgically (lymph node negative), bcl-2 overexpression remained a significant marker of good prognosis. This suggests that bcl-2 expression plays a crucial role in the regulation of apoptosis and disease progression in patients with operable cervical carcinoma. Greater understanding of the processes involved may lead to the development of improved medical treatment strategies.

Apoptosis occurs spontaneously in malignant tumours and its extent is enhanced by well established treatment modalities, such as irradiation, cytotoxic chemotherapy, heating, and hormone ablation. ' The regulation of this process is very complex and involves numerous genetic and epigenetic factors. Recent findings at the molecular level indicate that the interaction between certain oncogenes, such as c-myc, p53, bax, bcl-2 and its homologues, are mainly responsible for the regulation of spontaneous and treatment induced apoptosis. Probably the 
most prominant regulator of programmed cell death is the bcl-2 protein family. This family contains antiapoptotic proteins (for example, bcl-2, bcl- $\mathrm{X}_{\mathrm{L}}$ and $\mathrm{mcl}-1$ ) and proapoptotic proteins, such as bax. As a result of heterodimer (or oligomer) formation between bax and bcl-2 or other suppresors of programmed cell death, such as bcl- $\mathrm{X}_{\mathrm{L}}$ and $\mathrm{mcl}-1$, the actions of these proteins are neutralised. ${ }^{17}$ For oncogenes other than those in the bcl-2 family the regulation is sometimes so complex that it seems to be contradictory. For example, c-myc can stimulate mitosis or apoptosis, ${ }^{18} 19$ depending on the available stimuli. One of these stimuli (regulators) is bcl-2, which suppresses the cell death promoting function of c-myc without affecting c-myc mitogenic function. ${ }^{2021}$ Liang et $a l^{2}$ found that high levels of bcl-2 expression occurred in cervical carcinoma cells lacking functional p53. Silvestrini et $a l{ }^{23}$ Miyashita $e t a l^{24}$ and Liang et $a l^{2}$ all reported high levels of bcl-2 expression in p53 negative tumours. Haldar et $a l^{25}$ and Miyashita et $a l^{4}$ showed that overexpression of p53 can induce down regulation of bcl-2 expression at both the protein and mRNA level. This indicates that functional p53 can transcriptionally repress bcl-2. Human papilloma virus (HPV) infection, which plays a crucial role in cervical carcinogenesis, often leads to the inactivation of wild type $\mathrm{p} 53$ by HPV E6. ${ }^{26}{ }^{27}$ This may explain the important role of bcl-2 in the clinical behaviour of cervical carcinoma.

Bcl-2 transfection has been demonstrated to confer resistance to anti-cancer agents via a non-conventional drug resistance pathway ${ }^{28}$ In vivo and in vitro studies have demonstrated a role for the bcl-2 protein in the prevention of apoptosis induced by anti-cancer drugs and radiotherapy. ${ }^{29} 30$ Previous data from our laboratory ${ }^{14}$ and others ${ }^{31}$ strongly suggest that bcl-2 expression may be a predictor of response to chemotherapy or endocrine therapy in patients with breast cancer. There are several published observations about the parallel increase in bax concentrations opposing and neutralising the high level of bcl-2 expression in some transfectants and solid tumours, which may explain resistance to treatment by bcl- 2 negative and probably bax negative tumours. ${ }^{1732}$

Our results show a strong correlation between bcl-2 expression and prognosis in cervical carcinoma. The expression of other genes involved in the regulation of apoptosis in cervical cancer could, in future studies, shed more light on the understanding of the association between bcl-2 overexpression in primary tumours and beneficial clinical outcome.

1 Kerr JFR, Winterford CM, Harmon BV. Apoptosis: its significance in cancer and cancer therapy. Cancer 1994;73:2013-23.

2 Hockenbery D, Nunez G, Milliman C, Schreiber RD, Korsmeyer SJ. Bcl-2 is an inner mitochondrial membrane protein that blocks programmed cell death. Nature 1990;348:334-6.

3 Akao Y, Otsuki Y, Kataoka S, Ito Y, Tsujimoto Y. Multiple subcellular localization of bcl-2: detection in nuclear outer membrane, endoplasmic reticulum membrane, and mitochondrial membrane. Cancer Res 1994;54:2468-71.
4 Chen-Levy Z, Cleary ML. Membrane topology of the bcl-2 proto-oncogenic protein demonstrated in vitro. $f \mathrm{Biol}$ Chem 1990;265:4929-33.

5 De Jong D, Prins FA, Mason DY, Reed JC, Van Ommen GB, Kluin PM. Subcellular localization of the bcl-2 protein in malignant and normal lymphoid cells. Cancer Res 1994; 54:256-60.

6 Krajewski S, Tanaka S, Takayama S, Schibler MJ, Fenton W, Reed JC. Investigation of the subcellular distribution of the bcl- 2 oncoprotein: residence in the nuclear envelope, endoplasmic reticulum, and outer mithochondrial membranes. Cancer Res 1993;53:4701-14.

7 Monaghan P, Robertson D, Amos TAS, Dyer MJS, Mason DY, Greaves MF. Ultrastructural localization of bcl-2 protein. F Histochem Cytochem 1992;40:1819-25.

8 Shepherd JH. Cervical and vulva cancer: changes in FIGO definitions of staging. Br f Obstet Gynaecol 1996;103:405-6.

9 Kaplan EL, Meier P. Non-parametric estimation from incomplete observations. 7 Am Stat Assoc 1958;53:457-81.

10 Cox DR. Regression models and life tables. F R Stat Soc B 1972;34:187-220.

11 Sanders CA, Yano T, Clark HM, Harris C, Longo DL, Jaffe $\mathrm{ES}$, et al. p53 mutation is associated with progression in follicular lymphomas. Blood 1993;82:1994-2004.

12 Pezzella F, Turley H, Kuzu I, Tungekar MF, Dunnill MS, Pierce CB, et al. Bcl-2 protein in non-small-cell lung carcinoma. N Eng f Med 1993;329:690-4.

13 Baretton GB, Diebold J, Christoforis G, Vogt M, Müller C Dopfer K, et al. Apoptosis and immunohistochemical bcl-2 expression in colorectal adenomas and carcinomas. Cancer 1996;77:255-64.

14 Hellemans P, van Dam PA, Weyler J, Van Oosterom AT, Buytaert P, Van Marck E. Prognostic value of bcl-2 expression in invasive breast cancer. Br f Cancer 1995;72:354-60

15 Diebold J, Baretton G, Felchner M, Meier W, Dopfer K, Schmidt M, et al. Bcl-2 expression and apoptosis in ovarian

16 Levine EL, Davidson SE, Roberts SA, Chadwick CA, Potten CS, West CML. Apoptosis as predictor of response to radiotherapy in cervical carcinoma. Lancet 1994;344:472.

17 Miyashita T, Kitada S, Krajewski S, Horne WA, Delia D, Reed JC. Overexpression of the bcl-2 protein increas
half-life of p21

18 Evan GI, Wyllie AH, Gilbert CS, Littlewood TD, Land H, Brooks $\mathrm{M}$, et al. Induction of apoptosis in fibroblasts by c-myc protein. Cell 1992;69:119-28.

19 Shi Y, Glynn JM, Guilbert LJ, Cotter TG, Bissonnette RP, Green DR. Role for c-myc in activation-induced apoptotic cell death in T cell hybridomas. Science 1992;257:212-14.

20 Bissonette RP, Echeverri F, Mahboubi A, Green DR. Apoptotic cell death induced by c-myc is inhibited by bcl-2. Nature 1992;359:552-4.

21 Fanidi A, Harrington EA, Evan GI. Cooperative interaction between c-myc and bcl-2 proto-oncogene. Nature 1992; 359:554-6.

22 Liang XH, Mungal S, Ayscue A, Meissner JD, Wodnicki P, Hockenbery $\mathrm{D}$, et al. $\mathrm{Bcl}-2$ protooncogene expression in cervical carcinoma cell lines containing inactive p53. $f$ Cell cervical carcinoma cell lines

23 Silvestrini R, Veneroni S, Daidone MG, Benini E, Boracchi $\mathrm{P}$, Mezzetti M, et al. The bcl-2 protein: a prognostic indicator strongly related to p53 protein in lymph-node-negative breast cancer patients. $\mathcal{F}$ Natl Cancer Inst 1994;86:499-504.

24 Miyashita T, Krajewski S, Krajewska M, Wong HG, Lin $\mathrm{HK}$, Liebermann DA, et al. Tumor supressor p53 is a regulator of bcl-2 and bax gene expression in vitro and in vivo. Oncogene 1994;9:1799-805.

25 Haldar S, Negrini M, Monne M, Sabbioni S, Croce CM. Down-regulation of bcl-2 by $\mathrm{p} 53$ in breast cancer cells. Cancer Res 1994;54:2095-7.

26 Werness BA, Levine AJ, Howley PM. Association of human papillomavirus type 16 and 18 E6 proteins with p53. Science 1990;248:76-9.

27 Dyson N, Howley PM, Münger K, Harlow E. The human papilloma virus-16 E7 oncoprotein is able to bind to the retinoblastoma gene product. Science 1989;243:934-7.

28 Fischer TC, Milner AE, Gregory CD, Jackmann AL, Aherne GW, Hartley JA, et al. Bcl-2 modulation of apoptosis induced by anticancer drugs: resistance to thymidylate stress is independent of classical resistance pathways. Cancer Res 1993;53:3321-6.

29 Campos L, Rouault JP, Sabido O, Oriol P, Roubi N, Vasselon $C$, et al. High expression of bcl-2 protein in acute myeloid leukemia cells is associated with poor response to chemotherapy. Blood 1993;81:3091-6.

30 Miyashita T, Reed JC. Bcl-2 oncoprotein blocks chemotherapy-induced apoptosis in human leukemia cell line. Blood 1993;81:151-7.

31 Gee JMW, Robertson JFR, Ellis OA, Willsher P, McClelland $\mathrm{RA}$. Hoyle $\mathrm{HB}$, et al. Immunocytochemical localisation of bcl-2 protein in human breast cancers and its relationship to a series of prognostic markers and response to endocrine therapy. Int $\mathcal{F}$ Cancer 1994;59:619-28.

32 Krajewski S, Blomqvist C, Franssila K, Krajewska M, Wasenius VM, Niskanen E, et al. Reduced expression of proapoptotic gene BAX is associated with poor response rates to combination chemotherapy and shorter survival in women with metastatic breast adenocarcinoma. Cancer Res 1995;55:4471-8. 